\title{
La influencia de los discounter en el cambio de habitos de consumo de los colombianos
}

\author{
The influence of discounter in changing the consumption habits of colombians
}

DOI: 10.21803/adgnosis.v8i8.362

\section{Resumen}

Los discounters en los últimos 7 años de operación han intervenido en las decisiones de compra de los consumidores colombianos, los cuales son atraídos por los precios bajos de las marcas propias que cuentan con una calidad aceptable para ellos, en comparación de la competencia. De acuerdo a lo anterior los otros canales y formatos presentan perdida de la participación del mercado, sobre todo el canal tradicional (tiendas de barrio), los cuales han venido librando una batalla para ser sostenibles en el tiempo, pero, aun así, decreciendo el 14\% entre el 2013 y 2018. (Nielsen, 2019). Este modelo disruptivo de retail minorista está basado en 4 tácticas: surtido, área de ventas reducidas, logística y estructura organizacional horizontal; dándole eficiencias operativas diferenciándolos de los otros canales del mercado. Como resultados, se identificaron estas tácticas, se analizaron los modelo de operación basado en el marketing mix de Philip Kotler (4'Ps) y se reconocieron variables del mercado, como: el índice de deslealtad, el anhelo de aumentar el poder adquisitivo de compra (Matías Espósito, 2001) y consumidores híper informados causado por la transformación digital y globalización; lo cual permite comprender con mayor profundidad el crecimiento e influencia que este formato ha tenido en el mercado colombiano.

Palabras clave: Discounters, Hard Discount, tiendas de descuento duro, minorista, retail colombiano, canales de distribución.

\begin{abstract}
Discounters in the last 7 years of operation have intervened in the purchase decisions of Colombian consumers, which are attracted by the low prices of own brands that have an acceptable quality for them, in comparison to the competition. According to the above, the other channels and formats represent a loss of market share, especially the traditional channel (neighborhood stores), which have been fighting a battle to be sustainable over time, despite of it, decreasing the 14\% between 2013 and 2018. (Nielsen, 2019). This disruptive retail model is based on 4 tactics: assortment, reduced sales area, logistics and horizontal organizational structure; giving it operational efficiencies differentiating them from the other channels of the market. When identifying these tactics, analyze their operating model based on the marketing mix of Philip Kotler (4'Ps) and recognize market variables, such as: the disloyalty index, the desire to increase purchasing power (Matías Espósito, 2001) and hyper informed consumers caused by digital transformation and globalization; It allows us to know in greater depth the growth and influence that this format has had in the Colombian market.
\end{abstract}

Keywords: Discounters, Hard Discount, channels, Colombian retail, retailer, retail trader, retail dealer, distribution channels

\section{Sherly Hoyos Estrada ${ }^{1}$}

sherhe@hotmail.com

\section{Cómo citar este artículo:}

Hoyos, S. (2019). La influencia de los discounter en el cambio de habitos de consumo de los colombianos 8(8), p. 91-98. DOI: 10.21803/adgnosis.v8i8.362

1 Estudiante del MBA de la Universidad Tecnológica de Bolívar. , Orcid https://orcid.org/0000-0003-2533-5344 


\section{Introducción}

Los discouxnters, Hard discount o tiendas de descuento, son organizaciones que se destacan por tener ventas en sector retail gracias a características como surtido limitado, marcas propias, precios bajos y un control de costes, compiten en precio, calidad, consistencia y simplicidad. Adicionalmente, los descuentos aplican su enfoque sin adornos, los productos se exhiben a menudo en el suelo en palets y se preparan para la venta al por menor llegando a utilizar palets de medio tamaño para optimizar aún más el espacio en el suelo. El retail en Colombia es muy competido y está compuesto por diversos canales, tales como el tradicional (tiendas de barrio), independientes (Aliados Surtimax, Coratiendas, Red Contigo, Mercados Zapatoca) y moderno (supermercados, hipermercados y grandes superficies), entre otros.

Según el Índice Global de Mercado Minorista elaborado por A.T Kearney en el 2016 Colombia ocupó el segundo lugar a nivel Latinoamérica y la posición 15 a nivel mundial, este indicador analiza la proyección de desarrollo de los países para invertir en la industria del retail, esto demuestra el avance que ha obtenido el país, ya que en el 2007 se encontraba en la posición 30 (Katz Pinski \& Herrera Gámez, 2018). Este crecimiento se dio por el desarrollo de los canales: moderno e independientes, pero, sobre todo, por la aparición del nuevo canal de los Hard Discount o Discounters que desde el 2009 en Colombia iniciaron operaciones bajo el formato D1 “de uno" del Grupo Koba, posteriormente Tiendas Ara de la multinacional Jerónimo Martins y Justo \& Bueno del Grupo Reve.

En el 2013 este canal empezó a tener presencia en los hogares colombianos, al cierre del 2018 obtuvieron con una participación del $16 \%$ de todo el mercado colombiano, afectando al canal tradicional (tiendas de barrios) que contaban con el $48 \%$ en el 2013 y el 2018 finalizo con el $41 \%$, decreciendo el $14 \%$; otro canal afectado por los Discounters fue el de hipermercados de cadena que perdieron $8 \%$ de la participación durante los mismos 5 años de medición (2013 - 2018), es decir más del $50 \%$ de las ventas operativas que tuvieron en el 2013 (Nielsen, 2019).
Otro canal que se está fortaleciendo en el país es el Cash \& Carry, este formato consiste en grandes superficies que ofrecen presentaciones de mayor tamaño, reduciendo el gasto operativo tendiendo como bodega la estantería y generando descuentos por mayor número de unidades de una misma referencia, ejemplo de ello en Colombia es Pricesmart, Alkosto y Surtimayorista del Grupo Éxito, este último con su accionista mayoritario el Grupo Pão de Açúcar de Brasil que cuenta con la marca Assaí busca tener sinergias que pretenden desarrollar la marca desde el 2020 (Nielsen, 2019).

Es de esta manera como se observa que el retail minorista ha evolucionado en el país y que factores han intervenido para generar un cambio de hábito de consumo de los hogares colombianos, diversificando el mercado, es por esto que se abordara cómo está compuesto la distribución de este en Colombia, específicamente del canal Hard Discount y como a impacto la economía en general. Este formato ha aportado en un gran porcentaje en el desarrollo del retail, poniendo en riesgo notoriamente al mercado minorista tradicional (tiendas de barrio).

De esta forma es como se plantea inicialmente un trasfondo teórico en donde se observa como diversas investigaciones respecto al comportamiento del consumidor, muestran las tácticas de este retail minorista moderno por captar la atención de los compradores y como sus prácticas sencillas, pero eficaces, les han permitido a los consumidores aumentar su poder adquisitivo al gastar menos en el abastecimiento de sus hogares.

Seguidamente se aborda los Discounters como establecimientos de distribución minorista que vende productos con muy bajos precios, siendo un formato de supermercado de origen alemán. Este canal minorista ha tenido un crecimiento acelerado dentro de nuestro mercado con una alta densidad de número de tiendas, más 2.000 en Colombia con D1 "de uno" del Grupo Koba, Tiendas Ara de la multinacional Jerónimo Martins y Justo \& Bueno del Grupo Reve, 
obteniendo ventas mayores a 8.9 billones a finales del 2018, teniendo así una participación del $16 \%$ de todo el mercado colombiano, impactando al canal tradicional (tiendas de barrio) que por más de 50 años no perdía participación.

Finalmente, se analiza como la deslealtad del consumidor ha afectado los hábitos de consumo de la generación actual, con nuevos cambios culturales dentro de sus intereses por la compra de los productos de la canasta familiar.

\section{Trasfondo teórico}

Desde el punto de vista del comportamiento del consumidor, el origen de los estudios sobre este tema se encuentra en los primeros análisis microeconómicos de la conducta de los humanos. A partir de estos estudios, distintos autores expresaron durante la década de los 50 y principios de los 60 lo que se señaló teoría clásica del comportamiento del consumidor, caracterizada por su enfoque particularmente utilitarista (Henao \& Córdoba, 2007).

Dentro de la dimensión económica existe un ciclo formado por la producción, el intercambio y el consumo; este último es la actividad económica de mayor relación con la satisfacción de las necesidades, los artículos que el sujeto adquiere a través del consumo es la respuesta directa a la necesidad y estos mismos son la fuente que proporciona satisfacción. De este modo, la conducta de consumo es considerada un proceso de elección y se explica en función de la utilidad que los distintos bienes proporcionan a los sujetos (Giraldo López, 2007). En definitiva, la elección de los productos dependerá de las predilecciones de los consumidores, y se verá a su vez, limitada por la condición presupuestaria.

En el caso del retail en Colombia, fue donde los Discounters encontraron la oportunidad de captar clientes a través de sus tácticas y modelo de operación, ofreciendo a los consumidores productos de aceptable calidad con muy buen desempeño a precios bajos, satisfaciendo necesidades con la oferta de producto de sus marcas propias, exclusivas o blandas y valorando la condición económica de los consumidores entre- gándoles diferencias superior al 30\% en comparación de sus competidores o de otras marcas y canales.

\section{Metodología.}

Desde un enfoque descriptivo se pretende estudiar la influencia de los Dicosntuer en el cambio de hábito de consumo de los colombianos, para tales fines, se parte de una revisión de la literatura en donde se investigan trabajos vinculados con la temática en plataformas científicas como Scopus y Wos. Además se complementan los resultados de estos estudios con publicaciones y cifras de instituciones en Colombia que investigan en detalle los movimientos del sector retail, así como investigaciones e informes de marcas especializadas en recopilar este información y también desde las distintas ópticas, abarcando desde el consumidor, tenderos, distribuidores y grandes superficies del retail minorista, para finalmente, expresar conclusiones que permitan identificar elementos culturales en las familias del país que pueden generar cambios en los hábitos de consumo.

\section{Hallazgos}

Las tácticas de los Discounters, la clave de su éxito

En sus 10 años de operación en Colombia, los Discounters han impactado en las decisiones de compra de los consumidores colombianos, este modelo disruptivo de retail minorista, el cual fue creado desde 1946 en la Alemania de la post guerra, se ha convertido en toda una novedad para los consumidores colombianos. Al mismo tiempo se han identificado 4 tácticas utilizadas por este formato que lo diferencia de las otras cadenas y formatos.

\section{Surtido}

Los discounters fueron los primeros en innovar para optimizar el tiempo de surtir en lineales utilizando técnicas de PDQ (pallet deal quantity, por sus siglas en inglés) es la capacidad de exhibir los productos en los lineales en las mismas cajas de embalaje original.

Tener un surtido limitado es uno de los principios fundamentales de este formato y es un determinante de su éxito, por lo general cuentan entre 200 a 400 re- 
ferencias aproximadamente. No cuentan con grandes espacios para almacenamiento, por esta razón todo está exhibido en el piso de venta, permitiéndoles enfocar las negociones con los proveedores y reduciendo las opciones de los consumidores, como consecuencia les permite un manejo eficiente de la rotación de inventario, mientras que en el canal moderno encontramos formatos que manejan hasta 5000 referencias o más.

El surtido de los hard discount está compuesto del $90 \%$ en categorías básicas y el $10 \%$ restante en productos nuevos que no se consiguen en el canal tradicional, llamadas categorías destino, como: aceite de oliva, vinos, quesos especializados, carnes frías o salsas y aderezos, muchos de ellos apalancados en desarrollo de marcas exclusivas e importadas con el fin de generar innovación a los consumidores.

\section{Área de ventas reducidas}

Este formato ha realizado una expansión rápida dado que puede iniciar operación con áreas de venta de tamaño medio, entre 150 a 350 metros cuadrados, llegando a zonas de alta densidad residencial y comercial con menor inversión por apertura frente a la competencia.

Logística: utilizan técnicas de Cross Docking, la cual consiste que en los centros de distribución se consolidan los pedidos de diferentes proveedores sin almacenamiento y luego son despachados a las tiendas, esto les permite reducir significativamente los gastos logísticos teniendo mejores costos que la competencia, sobre todo, en comparación con el canal tradicional donde cada proveedor debe realizar entrega directa en las tiendas de barrio.

\section{Estructura organizacional}

Cuentan con una plantilla de personal notablemente reducida en comparación de su competencia, utilizando estructura organizacional horizontal, de modo que no tienen personal especializado, puesto que sus equipos están conformado entre 4 a 6 integrantes como máximo, donde cada uno de ellos puede realizar el trabajo de su compañero generando así trabajadores integrales que desempeñan diferentes servicios dentro del punto de venta, tales como: recibo de mercancía, surtir productos, caja de ventas e incluso mantener las labores de aseo y mantenimiento del establecimiento.

\section{Modelo de operación}

Para aclarar el modelo de operación de los Discounters aplicaremos el análisis del marketing mix, las 4Ps (Kotler \& Armstrong, 2013).

En el Producto, los discounters tienen como eje principal de su propuesta de valor ofertar productos de bajo costo a una calidad aceptable por el consumidor; optimizan los tiempos de pago y potencializan a los proveedores locales, al contar en su gran mayoría con marcas propias o marcas blandas tienen flexibilidad sobre la maquila, utilizando diversos proveedores en las diferentes regiones del país por el mismo producto.

Por parte del Precio, previo al origen de los discounters los consumidores no contaban con alternativas de productos al realizar sus compras y solo contaban con las marcas reconocidas a los precios que se manejaban en el mercado, que por lo general eran muy lineales o similares, en aquellos tiempos el diferencial era la presentación, en el canal tradicional están disponibles las de menor tamaño, sin embargo, ofrecían las mismas marcas. Los Discounters le dieron diversificación al mercado y a su vez a los hábitos de consumo de los colombianos, brindándoles productos de marcas alternativas a muy bajo precio, esto hizo reaccionar a las grandes superficies en el desarrollo de sus marcas propias para mantener la participación del mercado y en el caso de las tiendas de barrio no cuentan con musculo financiero o capacidad de compras volumétricas para desarrollar marcas propias.

Por su parte La Plaza, por muchos años el pilar de consumo de los colombianos ha sido el canal tradicional, las tiendas de barrio, por la cercanía que manejan los tenederos con su clientela en lo personal al conocer sus gustos y necesidades, facilidad de pago, el famoso "fiado", y la cercanía de estas con los compradores en distancia aumentando la probabilidad de compra.

Las grandes superficies también están librando 
una gran batalla con los Discounters para no perder participación en el mercado, realizan arriesgadas maniobras como tratar de combatir con sus marcas propias precio a precio con los discounters, reformar su propuesta de valor orientada a la experiencia de compra, como lo hizo el grupo Éxito con sus nuevos formatos de ÉxitoWOW, Carulla Freshmarket y Carulla Smartmarket, considerados según América Retail como los mejores formatos de innovación en el 2019 (América Retail, 2009), otra medida usada por el canal moderno para contrarrestar el efecto de los Discounters fue abriendo más sucursales en formatos pequeños como lo hizo Olímpica en la costa Atlántica intentando crear cercanía con el consumidor.

Los discounters tuvieron una rápida penetración en el mercado por la alta densidad de aperturas de puntos de venta en toda la extensión del país, liderado por D1 con 1300 tiendas en 243 municipios, seguido de Justo \& Bueno con 1000 y por último Ara con 600 (González Bell, 2020) generando al consumidor cercanía y posicionamiento de marca visual, puesto que los consumidores perciben que en cada barrio por lo menos hay una tienda de este formato.

$\mathrm{Al}$ cierre del primer semestre del 2019 la mayor participación en ventas del canal de los Discounters se lo reparten entre D1, que cuenta con $50,3 \%$ seguido por Ara que tiene el 23,2\% y finalmente Justo \& Bueno con el 10,7\%. En el caso de D1 tiene una presencia en los hogares colombianos del $55 \%$, y mantiene una frecuencia de los clientes de 13 días, así como un ticket promedio por cliente de $\$ 24,500$; siendo esta marca la primera en tener operaciones en Colombia en este nuevo canal (Neira Marciales, 2019).

Actualmente hay una gran variedad de productores en el mercado con marcas nuevas que no tienen comunicación en medios masivos y a pesar que son de buena calidad no son conocidos por los consumidores, es cuando ven atractivo utilizar este formato para la colocación de sus productos en los hogares colombianos, por la cobertura y densidad de puntos de ventas.

Finalmente, la Promoción, en esta dimensión cuentan con precios por debajo de los otros canales y formatos, aproximadamente entre el $30 \%$ al $35 \%$, esto se debe a su bajo costo de operación. Los consumidores cada vez quieran más promociones o descuentos debido a la reducción de ingresos, altos impuestos e inflación, esto hace que sean consumidores de compra conveniente y conformista, de acuerdo con los cálculos de Raddar, en el año 2010, el comprador de promociones era el $17 \%$, el de oportunidad era el $40 \%$ y el de valor era el $42 \%$, al cierre del 2018, el $90 \%$ del mercado era comprador de promociones (Portafolio, 2018).

Sus tácticas y modelo de operación fueron los pilares para que los Discounters crearon una propuesta de valor tomando lo mejor de cada formato (canal tradicional y moderno), como son presentaciones ajustadas (medianas), un modelo de exhibición rápido, alta densidad de puntos de ventas para generar cercanía con los consumidores, gran participación de marcas propias, blandas y exclusivas, negociación de compra directa con los proveedores, infraestructuras de venta con inversión menor, gasto de personal reducido y confort al momento de la compra, que les permite trasladarle el costo bajo al consumidor en el precio de venta (Chocontá, 2017).

\section{La deslealtad de los consumidores}

El consumidor acude a otras marcas por la necesidad de aumentar su poder adquisitivo de compra, es la contradicción del Brand Equity, ya que los consumidores quieren los productos, pero no pueden pagar por ello (Matías Espósito, 2001). Los niveles de deslealtad por parte de los consumidores han venido en aumento los últimos años, según el Estudio Global de Nielsen sobre Lealtad del Consumidor 2019, solo el 7\% de los colombianos participantes se consideraron leal a sus marcas favoritas, un $40 \%$ de los colombianos les encanta probar activamente nuevos productos y marcas, y un $47 \%$ de los consumidores colombianos son exploradores activos a probar marcas nuevas. Los factores de elección son en marcados en dos aspectos, relación calidad-precio con un $24 \%$ y precios y promociones con otro $24 \%$, los factores de éxito de las marcas nuevas donde los consumidores consideran con mayor relevancia para elegir un producto de otro, según el estudio, obedece al $24 \%$ calidad funcio- 
nal superior y otro $24 \%$ en la facilidad y conveniencia, factores claves que los Discounters entendieron para captar y satisfacer las necesidades de su clientela (Nielsen, 2019).

Como afirma Fedesarrollo (2019) quien analiza el índice de confianza del consumidor (ICC), el balance promedio del ICC fue de $-7,4 \%$, lo que presenta una reducción de 6,9pps con respecto al balance promedio de 2018 (-0,5\%). En diciembre de 2019, el Índice de Confianza del consumidor registró un balance de $-9,5 \%$, lo que representa un aumento de 4,9 pps frente al mes anterior $(14,4 \%)$. Este resultado obedeció principalmente al incremento en el Índice de Expectativas del Consumidor ( $+7,8 \mathrm{pps}$ ) y en menor medida al aumento en el Índice de Condiciones Económicas (+0,6pps) (Fedesarrollo, 2020).

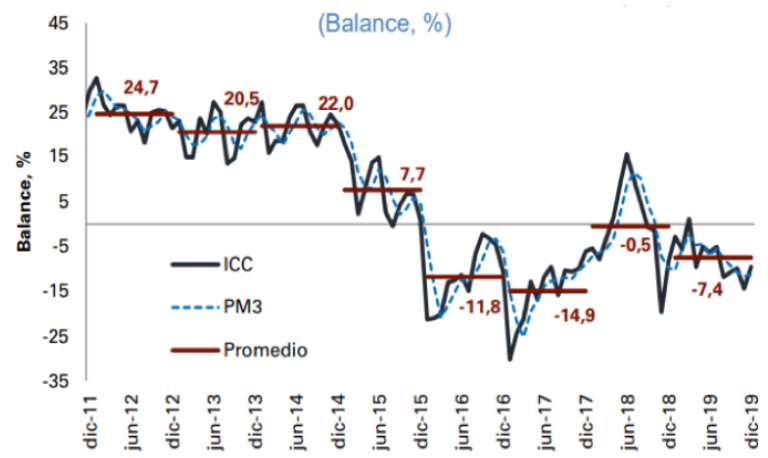

Figura 1. Índice de confianza del consumidor 2019.

Fuente: Fedesarrollo (2020).

Este índice en terreno negativo obedece a las desconfianza de los consumidores influenciados por las reformas tributarias, tasa de desempleo, escándalos de corrupción y controversias en el gobierno; creándole al consumidor incertidumbres al momento de gastar en la industria manufacturera e industria de fabricación de productos farmacéuticos que obtuvieron una variación del -1,5\%, que por el contrario, las ventas reales del comercio minorista, las ventas reales sin combustibles y las ventas reales sin combustibles ni vehículos aumentaron $4,4 \%, 4,7 \%$ y $9,7 \%$, respectivamente (Fedesarrollo, 2020).

Este desarrollo en el comercio minorista ha sido impulsado en gran medida por los Hard Discount, que al cierre del 2018 tuvieron $\$ 8,9$ billones de ventas totales con un crecimiento exponencial del 510,7\% desde el 2013 que tenían ventas de $\$ 1,47$ billones. Según cifras de Euromonitor, realizó una proyección de la venta de los Discounters y se espera que para el 2023 tengan ventas de $\$ 25$ billones. (Neira Marciales, 2019).

Muchos consumidores han asumido muy bien el formato de autoservicio, donde en el primer Q del 2018 el 95\% de los hogares colombianos conformados entre 2 o 3 miembros, alguna vez han comprado en este formato con una frecuencia promedio de cada 4 días y un ticket promedio de $\$ 35.000$ COP, este estudio comprenden canales independientes, discounters, cash \& carry y supermercados de cadena. (Nielsen, Nielsen, 2018).

El perfil de los consumidores ha migrado por la transformación digital y globalización mundial, actualmente son hiperinformados, con diversidad de opciones y nuevas experiencias disponibles, teniendo cambios abruptos en los hábitos de consumo, donde el motivador mayor es el precio-calidad, los consumidores se sienten atraídos por el precio, pero a largo plazo la tendencia serán los rasgos tangibles de los productos, separándolos de la marca, que mantendrán la recompra de los consumidores, es decir, que se valoraran las características físicas del productos tales como: calidad, función y conveniencia. (Nielsen, Nielsen, 2018).

En la actualidad las oportunidades para captación de clientes a través de precio son más tangibles que nunca, los competidores en el retail consideran relevante tener estanterías bien abastecidas, diversidad de opciones de productos, precios, presentaciones y hasta diferentes plataformas en sus puntos de ventas para compras, (online - offline), según Nielsen consideran 5 aspectos claves para captar la atención de los consumidores y fomentar la deslealtad: aceptar la adicción a lo nuevo, adquisición de productos locales, interacción con los consumidores, compromiso a través de conveniencia y calidad, y captar al tráfico de clientes dentro el punto de venta. (Nielsen, Nielsen, 2019). 


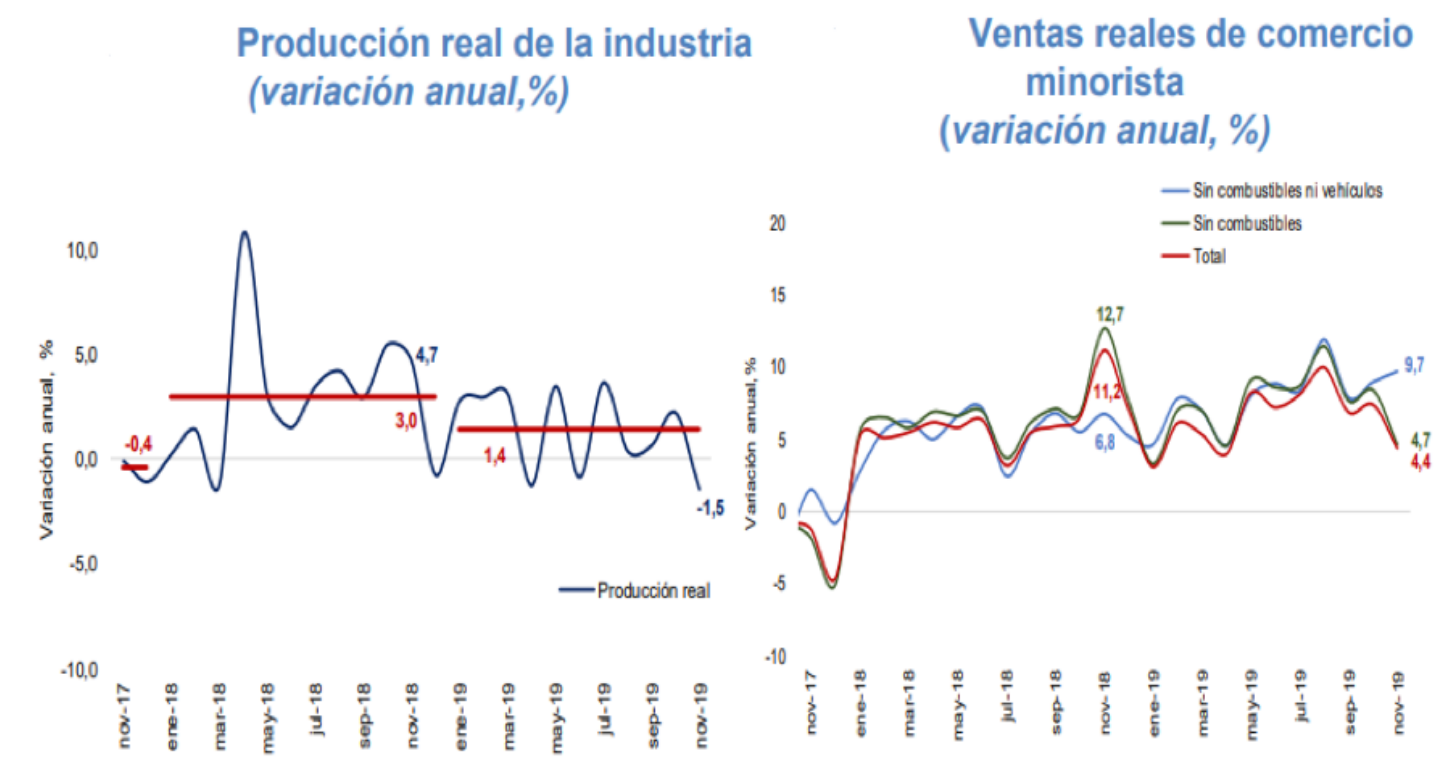

Figura 2. Variación producción real de la industria y de comercio minorista.

Fuente: Fedesarrollo (2020).

\section{Conclusiones}

Los discounters crearon un modelo disruptivo en el retail colombiano impactando al consumidor en sus hábitos de consumo dándoles alternativas en diversidad de marcas y tipos de productos con fácil acceso con el propósito de atraerlos.

Los consumidores han adoptado y aceptado muy bien el formato, puesto que han migrado a formato de autoservicio de conveniencia (cercanos) atraídos por este formato de precios bajos, otras condiciones que han impactado los hábitos de consumo han sido las condiciones internas del país como la incertidumbre del gobierno, bajos ingresos, impuestos altos y eventos de corrupción, convirtiéndolo en un consumidor analítico, pues que estudia todas las características de los productos más tangibles, precio-calidad. También se han visto influenciados por variables internacionales como la transformación digital y globalización, permitiéndoles tener información de primera mano de productos, marcas y mercado, sea para chequeo precios o validación desempeño de productos por comentarios de otros consumidores.

Este formato tiene gran proyección de crecimiento para los siguientes años, $30 \%$ de la participación total del mercado colombiano para el 2023 según (Neira Marciales, 2019), afectando en gran medida a la tienda de barrio en el canal tradicional, a estar en desventaja competitiva en colocación de productos de marcas poco reconocidas puesto que no cuentan con poder de negoción volumétrica por la falta de flujo de caja o financiación, en definitiva si el canal tradicional no desarrolla estrategias para aumentar la captación de clientes, fidelización y frecuencia de compra; su tendencia seguirá siendo decreciente en la participación del mercado. 


\section{$\stackrel{\sim}{N}$ Referencias}

Chocontá, F.A. (2017). Impacto del modelodetiendas hard discount en Colombia. http://hdl.handle.net/10654/16621.

Fedesarrollo. (2019). Tendencia Económica. Editorial: Actualización de las perspectivas económicas 2020-2021, (208), $1-38$.

Fedesarrollo. (20 de enero de 2020). Índice de Confianza del Consumidor (ICC). https://www.repository.fedesarrollo.org. co/handle/11445/3870?locale-attribute=en

Giraldo López,J.(2007). Comportamiento del consumidor.https:// www.gestiopolis.com/comportamiento-del-consumidor/

González Bell, J. (9 de enero de 2020). Las movidas que hicieron los discounters en 2019 y los planes de expansión para 2020. https://www.larepublica.co/empresas/las-movidas-quehicieron-los-discounters-en-2019-y-los-planes-de-expansionpara-2020-2949460

Henao, O., \& Córdoba L. J. (2007). Comportamiento del consumidor, una mirada sociológica. Entramado, 3(2), 18-29.

Katz Pinski, D., \& Herrera Gámez, V. (2018). Las tiendas Hard Discount: nuevo jugador en el mercado. Colegio de Estudios Superiores de Administración-CESA.

Kotler, P., \& Armstrong, G. (2013). Fundamentos de Marketing. México: Pearson Educación.

Matías Espósito, I. (2001). Brand Equity: Modelos de Valuación (Tesis de maestría). Universidad del CEMA, Buenos Aires, Argentina.

Neira Marciales, L. (5 de agosto de 2019). Dl alcanzó 50,3\% del mercado de discounters seguido de Ara $(23,2 \%)$ y Justo y Bueno $(10,7 \%)$. https://www.larepublica.co/empresas/dl-alcanzo503-del-mercado-de-los-discounters-seguido-de-ara-232-yjusto-y-bueno-107-2892887

Nielsen. (2018). Redefiniendo la conveniencia, una creciente necesidad del consumidor. https://www.nielsen.com/co/es/ insights/article/2018/redefiniendo-la-conveniencia-unacreciente-necesidad-del-consumidor/
Nielsen. (2019). La deslealtad del consumidor: la nueva realidad. https://www.nielsen.com/co/es/insights/2019/la-deslealtaddel-consumidor-la-nueva-realidad/

Nielsen. (2019). Cash \& Carry: Un formato por descubrir. https:// www.nielsen.com/co/es/insights/article/2018/cash-andcarry-un-formato-por-descubrir/

Nielsen. (2015). Principales tendencias del mercado de alimentos. https://www.nielsen.com/co/es/insights/article/2015/ tendencias-alimentos-colombia/

Nielsen. (2018). Hard Discount y Cash \& Carry impulsan la importancia del autoservicio en Colombia. https://www. nielsen.com/co/es/insights/article/2018/hard-discount-ycash-and-carry-impulsan-la-importancia-del-autoservicio-encolombia/

Nielsen. (2019). Tendencias del consumo en Colombia - abril 2019. https:/www.nielsen.com/co/es/insights/article/2019/ tendencias-del-consumo-en-colombia-abril-2019/

Portafolio. (15 de marzo de 2018). El colombiano se ha convertido en comprador conveniente $\mathrm{y}$ consumidor conformista. https://www.portafolio.co/economia/el-colombiano-seha-convertido-en-comprador-conveniente-y-consumidorconformista-515275

Retail, A. (10 de octubre de 2019). América Retail. https://www. america-retail.com/colombia/colombia-el-retail-en-el-quecreo-caso-carulla-freshmarket/

Retail, A. (15 de julio de 2019). América Retail. https://www. america-retail.com/opinion/grupo-exito-nominado-comomejor-e-tailer-de-latinoamerica-retail-hall-of-fame/

Rodríguez, G. (19 de noviembre de 2019). La resiliencia de las cadenas de supermercados. https://www.nielsen.com/co/ es/insights/article/2019/la-resiliencia-de-las-cadenas-desupermercados/ 